\title{
Flow and the dynamics of conscious thought
}

\author{
Joshua Shepherd ${ }^{1,2}$ (D)
}

Accepted: 25 July 2021 / Published online: 21 August 2021

(c) The Author(s) 2021

\begin{abstract}
The flow construct has been influential within positive psychology, sport psychology, the science of consciousness, the philosophy of agency, and popular culture. In spite of its longstanding influence, it remains unclear [a] how the constituents of the flow state 'hang together' - how they relate to each other causally and functionally- $-[b]$ in what sense flow is an 'optimal experience,' and [c] how best to describe the unique phenomenology of the flow state. As a result, difficulties persist for a clear understanding of the flow state's structure and function. After explicating the standard view of the flow construct (section one), I articulate several basic questions regarding its nature and functional roles (section two), and I argue that these questions are best answered by integrating flow within broader streams of research on the dynamics of thought, on cognitive control resource allocation, and on creative thought (sections three and four).
\end{abstract}

Keywords Flow $\cdot$ Creative thought $\cdot$ Mind wandering $\cdot$ Cognitive control · Dynamics of thought

\section{Introduction: the flow construct}

In this paper I explore the nature of 'flow', a construct that has been influential within the positive psychology movement (Nakamura \& Csikszcentmihalyi, 2014) and within sport psychology (Chavez, 2008; Swann et al., 2012) (where it is often treated as synonymous with 'being in the zone'), to a lesser extent within the science of consciousness (Vuorre \& Metcalfe, 2016) and the philosophy of agency (Dreyfus, 2005), and to a larger extent in popular culture (see the recent PIXAR film Soul). Consider the following description of a flow state.

Joshua Shepherd

joshua.shepherd@carleton.ca

1 Carleton University, 1125 Colonel By Dr, Ottawa, ON K1S 5B6, Canada

2 University of Barcelona, Gran Via de Les Corts Catalanes, 585, 08007 Barcelona, Spain 
'So when I'm in the zone and, you know, I'm blocking out everything and I'm just in that game... It's almost like a trance, it's like a dreamlike state. It's almost as if time slows down. The ball doesn't seem to be going that fast anymore. It's a great feeling'.

That is a quote from Steve Zahler, owner of Modern Pinball NYC, about finding flow while playing pinball. It comes from a short video on flow by NBC Left Field 1 — one of dozens of such videos that any internet search for 'flow' or 'being in the zone' will turn up. How accurate is Zahler's description of flow? Interestingly, Zahler, seemingly off the cuff, accurately articulates a number of the features flow psychologists have identified as characteristic of the flow state. As typically elucidated-see, e.g., Jackson and Marsh (1996); Jackson et al. (1998); Nakamura and Csikszentmihalyi (2014); Swann et al. (2012); Marty-Dugas and Smilek (2019)_ the flow construct contains at least nine separable elements.

First, in the circumstances surrounding the agent's action, there is a balance between the agent's abilities or skills and the difficulty of the tasks in which the agent is engaged; in particular, the agent's skills are well-matched to the situation such that the relevant tasks are neither too challenging nor too easy.

Second, the agent's goals for action in the circumstances at hand are relatively clear.

Third, the situation affords clear feedback regarding action success and progress.

Fourth, the agent is in a state of concentration, or (more or less) fully absorbed attention.

Fifth, the agent experiences what researchers characterize as a merging of action and awareness. Csikzscentmihayi (2014) expands on this notion as follows.

Perhaps the clearest sign of flow is the experience of merging action and awareness. A person in flow does not operate with a dualistic perspective: one is very aware of one's actions, but not of the awareness itself. A tennis player pays undivided attention to the ball and the opponent, a chess master focuses on the strategy of the game, most states of religious ecstasy are reached by following complex ritual steps, yet for flow to be maintained, one cannot reflect on the act of awareness itself. The moment awareness is split so as to perceive the activity from "outside", the flow is interrupted $(2014,138)$.

Sixth, the agent does not experience themselves in a certain way-there is said to be a loss of reflective self-consciousness, or, as Nakamura and Csikszentmihalyi explicate this notion, a 'loss of awareness of oneself as a social actor' $(2014,240)$.

Seventh, the agent experiences a heightened sense of control over their actions and over their action options.

Eighth, the agent's experience of time is distorted-time seems to pass more quickly.

Ninth, the activity in which the agent is engaged is experienced as intrinsically rewarding.

\footnotetext{
1 Video available at https://www.youtube.com/watch?v=PXVoKrcrbPI, accessed March 29, 2021.
} 
Taken together, this is an interesting and fairly esoteric collection of properties to attribute to a single mental state or process. We are told that as attention is fully captured by features of a task, the self diminishes, action takes over awareness, the sense of time is bent, and at the same time we are told that one's sense of control increases. We are also given a characterization of this collection of events as normative for the brain and for the agent-a major claim of Csikszcentmihalyi's over the years has been that the flow state is an 'optimal experience,' something agents do and should try to re-enter, and something that contributes to the good life.

However, in spite of evident interest in the nature of flow across research paradigms in psychology and philosophy, and across popular culture, it remains unclear [a] how the constituents of the flow state 'hang together'-how they relate to each other causally and functionally-[b] in what sense flow is an 'optimal experience,' and [c] how best to describe the unique phenomenology of the flow state. As a result, difficulties persist for a clear understanding of the flow state's structure and function. In the next section I articulate three sets of pressing questions-questions for which I take it that we lack consensus answers. In section three I introduce three research streams - on the dynamics of thought, on cognitive control resource allocation, and on creative thought - that, I argue, can bear fruitfully on the study of flow. In section four I discuss ways research that places flow alongside these other constructs, and utilizes tools and ideas from these research streams, can make progress on the questions I articulated in section two.

\section{Questions about flow}

The aim of this section is to articulate several questions about flow. Since I take it [a] that many of these questions are open given current knowledge, [b] that future research could fruitfully bear on these questions, and [c] that a complete (and to some extent coherent) understanding of the flow state awaits a better grasp of how to answer these questions, I will not pretend to have failsafe answers to many of these questions, nor will I even attempt to answer many of them. The value of this section is in the articulation of the questions.

I will group the questions into clusters of three.

\subsection{How do the nine items said to make up the flow construct hang together?}

One option is that all nine items are necessary for flow to be present. For several reasons, this option is no good. First, although Csikszcentmihalyi and co-authors often discuss all nine items together, many treatments of flow, and many experiments, do not (see Marty-Dugas \& Smilek, 2019 for a discussion of this point). So flow science does not seem to take this option seriously. Second, many-arguably, all_-of the nine items are gradable in the sense that they can hold for an agent to greater or lesser degrees (one might wish to think of the loss of reflective self-consciousness as non-gradable). With so many gradable items on the list, how is one supposed to answer whether they are present or not in a particular 
case? The question becomes whether they are present to a sufficient degree. And it seems implausible, and unsupported by evidence, to think that flow can only be present if all nine items are to some arbitrary degree.

A second option is that, while we can find flow states without some of the nine items on this list, other items are necessary for the emergence of flow, or constitutive of the flow state. One can imagine any of the nine, or any combination of the nine, as necessary or constitutive - this we can largely leave as an exercise for the reader. But it is worth mentioning a recent proposal by Marty-Dugas and Smilek (2019) that we should reconceptualize flow primarily in terms of absorption.

In contrast to the common view that flow is comprised of a combination of nine facets, we wish to advocate for the position that the experience of flow is best characterized by a single-core concept, namely, the subjective experience of "deep, effortless concentration". By the term "deep" we mean the general experience of being fully engaged or completely focused on whatever one is doing; and by the term "effortless" we meant the act of sustaining one's attention without 'forcing it' or experiencing strain while doing so (2019, 1761).

I have no strong objection to a research program that focuses on absorption, or effortless concentration. Certainly, this seems to be a key facet of flow. But what is involved in thinking of this as the core of flow? Marty-Dugas and Smilek argue that reconceptualizing flow in this way has three advantages: it renders flow more specific, allowing for 'more targeted measurement,' it affords a clearer distinction between precursors, constituents, and consequences of flow, and it better allows for the measurement of flow for 'internal' tasks involving mental rather than bodily action.

I agree with Marty-Dugas and Smilek that clarity regarding items within the flow construct is essential. There is a real problem of cross-talk in the flow literature, and a serious worry that many papers discussing 'flow' are discussing very different things. In some cases, one finds researchers who ask for self-reports of all nine items, and then average over self-reports to get a score for flow. In other cases, some subset of the nine items is in view, and others are not mentioned. In still other cases, very different approaches are taken. So, for example, Swann et al. (2017) had participants elaborate freely on their experiences while performing tasks. As they write,

When optimal psychological states were reported, participants were asked if they were familiar with terms flow/"letting it happen" and/or clutch/"making it happen". If they answered affirmatively, they were asked to describe their understanding of those terms and explain how the identified state corresponded. The interviewer then judged whether they were referring to flow or clutch (as defined by the research team) (382).

The qualitative methods flow researchers often deploy sometimes force latitude in how the flow construct is understood by participants. This may, of course, present a problematic feedback loop, if ideas about flow in popular culture depart 
from the construct that drives theorizing in much psychology. At minimum, greater care is needed in generalizing from studies on some aspects of the flow construct to studies on other aspects.

While Marty-Dugas and Smilek's push towards greater clarity is welcome, however, I have two complaints about a sole focus on deep, effortless concentration. First, it is not clear why effortless concentration is any easier to measure than other items in cases of mental action. Second, the clearer distinction between precursors, constituents, and consequences is promising, but questions remain about how deep, effortless concentration relates to other items in the original flow construct. If these bear any significant relationships, then these questions should be pursued, and reconceptualizing flow in this way will be of little use.

It is possible, after all, that though some of the items in the original flow construct are inessential for understanding the preconditions or functions of flow, some of these items cluster in interesting ways. Perhaps the flow state is something like a 'homeostatic property cluster' (Boyd, 1991) in the sense that items within the flow construct as presently understood reliably correlate with each other given certain causal factors. If so, these items would carry inductive significance, and would raise questions about the mechanisms underlying these items that careful experiments could address.

In this connection, note that many researchers (e.g., Nakamura \& Csikszentmihalyi, 2014; Ulrich et al., 2018) treat the first three items as causal preconditions, and the last six as more or less constitutive of the flow state. Cochrane (2017) similarly conceptualizes the last six items as 'descriptive statements about what it is like to experience flow' (135), but notes that the absorption of attention may have both descriptive and generative aspects. This seems like a fruitful way to hypothesize a structure amongst these nine items, and it raises interesting questions.

Can manipulations within items one, two, or three (or four) produce noticeable differences in the remaining items? Are these items sufficient to explain the generation of all aspects of flow, or is the flow construct missing something? Note, in this connection, Cochrane (2017) point that greater attention to the agent's intention, the agent's performance, and relationships between these is needed to understand features like diminished self-consciousness and 'the sense of effortless control' (2017, $135)$.

Is it possible to generate flow-like effects solely in virtue of goal clarity or feedback clarity, or is the skill/difficulty balance essential? Conversely, how much degradation of feedback clarity or goal clarity is required to undermine flow-like effects?

How closely do items four through nine hang together, and what conditions (including individual differences) might mediate differences in their occurrence across various action contexts?

\subsection{What is the sense in which flow is an optimal experience?}

I will consider two possible answers to this question-both of which Csikszentmihalyi and co-authors seem, at different places, to have in view. 
On the first answer, flow is an optimal experience in the sense that it enhances well-being. Certainly, the flow state seems to have clear links to things that many people value-e.g., pleasure and achievement. But assessing flow's impact upon well-being depends not only upon the nature of the flow state-which I am presently putting in question-but upon theoretical disputes about the nature of wellbeing. These theoretical disputes differ in complicated ways across psychology and philosophy (see Bishop, 2015), and different theories would view the potential contributions of the flow state in very different ways. So whether, and in what way, flow might contribute to well-being is well beyond this paper's scope.

A second answer-not in competition with the first-is that flow in some activity enhances performance on that activity. This is a claim sometimes found in the scientific literature. So, for example, Nakamura and Csikszentmihalyi claim that 'when in flow, the individual operates at full capacity' (2014, 240). De Manzano et al. (2010) are more careful, claiming only a correlation: 'Expert performance is commonly accompanied by a subjective state of optimal experience called flow' (301).

Does flow actually enhance performance? There are reasons to be skeptical. Kennedy et al. (2014) found that an increase in reports of flow correlated with the metacognitive feeling that performance was good, even when performance was not good. If flow itself tracks metacognition for performance, then cases of misfiring metacognition may provide cases of flow in the absence of quality performance. Further, while an unpublished (as of yet) meta-analysis by Harris et al. (2020) found a small to moderate relationship between flow and performance enhancement, the authors report little evidence for direction of causation from flow to performance.

In my view, it would not be too surprising if flow and performance turn out to be only loosely related. This is because different activities allow many routes to success. Some activities permit, or even encourage, a kind of exploration that results in errors and on-the-fly corrections-the kinds of things that would not correlate strongly with flow. Consider, for example, improvisational actions. While some improv performers report something like flow states when improvising, the flow state tends to come and go, and this may be crucial to high-quality improvisation. Expert improvisers often cycle through periods of free-form play and exploration, as well as periods of planning and highly engaged cognitive control (Beaty, 2015). Some activities permit success even when goals and/or feedback are not clear or not readily available. Other activities demand persistence through difficulty, tolerance of challenges, or high input of effort. For such activities, seeking to stay in flow, rather than seeking out challenges or circumstances that push one to improve one's level of skill, might be a good way to remain mediocre.

Barbera Montero has offered a similar criticism, noting that peak experience and peak performance 'might not always line up' (Montero, 2016, 185). This is because, Montero argues, expert actions are not necessarily intrinsically rewarding, 'but are performed because they might lead to a future reward' (185). In addition, the presence of self-awareness is critical for at least some action types: pondering questions about what one is doing 'seems to be a very important part of many forms of expertise, such as those that encompass actions that may need to be justified... [for example] Repeatedly questioning the necessity of our 
actions... and critically evaluating the reasons for carrying them out is helpful in creating a good piece of writing, and perhaps this is why writing is particularly un-autotelic' (185).

Further, work that explicitly seeks to address the flow-performance question runs into a difficult potential confound. If this work seeks to measure the impact of the flow state, then it has to reliably produce the flow state. And if producing the flow state requires placing the agent into conditions in which her skill is well-matched to the difficulty, then it is easy to think that the same conditions that produce the flow state are those that explain enhanced performance.

One line of work that suggests a way forward explores links between mindfulness training and flow experience. There is some evidence, for example, that mindfulness training increases the emergence of flow states in sport performance (Aherne et al., 2011; Scott-Hamilton et al., 2016). If it could be demonstrated that the enhancement of flow states via mindfulness training also enhances performance, we might have some (obviously, only tentative) reason to think that flow enhances performance. But such work tends to ignore the relationship between flow state enhancement and performance enhancement. Moreover, a recent meta-analysis examined links between mindfulness training and performance enhancement (without examining flow state), and found some positive influence of mindfulness training on performance, but only for a limited group of sports. Shooting and dart throwing showed performance enhancement, but not cycling, hammer throwing, hurdling, judo, rugby, or running (Bühlmayer et al., 2017).

At present, the relationship between flow and performance is unclear.

\subsection{What kinds of conscious states are essential to flow?}

Considering this question places us in difficult territory. For, while the flow construct is built in part around a series of claims regarding phenomenology-that is, what it is like subjectively for someone to be in a flow state-the specifics of phenomenology have never generated much agreement in psychology or philosophy, and remain the subject of controversy.

One key part of the phenomenology - absorption, or effortless concentrationcan be understood as, in part, to do with attention. Whether the absence of effort is a critical part of absorption or not is, to my mind, unclear. Perhaps talk of 'effortless concentration' is one way of describing the agent's global state, as opposed to the way their attentional states feel at any given time.

Consider, further, the relationship between action/awareness merging and diminished self-consciousness. It is, to my mind, unclear to what degree these can be kept separate. Here is how Csikszentmihalyi describes action/awareness merging:

A tennis player pays undivided attention to the ball and the opponent, a chess master focuses on the strategy of the game, most states of religious ecstasy are reached by following complex ritual steps, yet for flow to be maintained, one cannot reflect on the act of awareness itself. The moment awareness is split so as to perceive the activity from "outside", the flow is interrupted $(2014,138)$. 
But arguably the act of reflecting on the act of awareness itself, the split within perception that Csikszentmihalyi talks about, involves (at least implicit) awareness of self. It may be, then, that diminished self-consciousness and action/awareness merging are two ways of describing the same thing. Note, incidentally, that Csikszentmihalyi et al. (2005) explicitly link these two items.

What are we to think about claims that time passes more quickly while in flow? Some researchers have found that this aspect is comparatively rare in flow-like conditions (Swann et al., 2012). Perhaps time distortion occurs only when absorption is near total. Something like this is suggested by Csikszentmihalyi et al. (2005) treatment of this item. As they have it, flow-like conditions so occupy attention (leading to, e.g., action/awareness merging) that attentional resources are diverted from time perception. This suggests a non-phenomenological reading of this item. Agents in flow may be disposed to judge that time has passed more quickly, even if their experience of time passing is, at any given moment, no different than it is in non-flow conditions. With that said, it would be interesting if rigorous exploration of flow phenomenology revealed a distortion in the experience of time passing.

The heightened sense of control does seem to be a genuine and separable aspect of phenomenology. And it would be interesting to see work that explored this sense more completely. One relevant question is how this sense of control relates to the more studied 'sense of agency'. A further question is whether the sense of control is a product of metacognition about action (see Vuorre \& Metcalf, 2016). What does the sense of control have to do with a sense of confidence regarding one's action or action options one is considering? Might they be the same thing?

Finally, consider the intrinsically rewarding aspect of flow. Is this separable from the other phenomenological elements, or does it supervene on at least some of them? There is, of course, a potential explanation for the positivity of flow that has little to do with these other elements. In the flow state, one tends to receive clear, positive feedback about the actions one performs. A constant influx of positive feedback might produce positive emotions (for related work, see Reber et al., 2004).

One also wonders about the relationship with absorption. Is absorption, or effortless concentration, an intrinsically positively valenced aspect of experience, or is it possible for concentration to have a negative (or a neutral) valence? I suspect that it is possiblejust imagine being completely immersed in an experience of fear-but this possibility is not much discussed in the flow literature, and it should be explicitly studied. ${ }^{2}$

Although the qualitative research methods that flow scientists often use have produced a wealth of interesting data regarding the experiences that surround the flow construct, differences in deployment of the flow construct across research paradigms make answering the above questions difficult. Granted, not all of these questions are pressing. But an understanding of experiential aspects of the flow state seems to require, at least, an understanding of how these elements relate to each other. I

\footnotetext{
2 A referee notes an interesting connection here with Debus's (2015) argument that a sufficient condition for the intrinsic value of a subject's activity is that the subject give the activity their full attention. Debus's argument runs through a claim that the experience of full attention is very fulfilling for the subject, so research that demonstrates that full attention can have negative valence would probably constitute a challenge to Debus's view.
} 
would emphasize the importance of understanding how experiences of absorption, of effortlessness, of heightened control, and of positivity hang together or come apart across different conditions.

\subsection{Summary of questions}

It may be useful to pull together here what I take to be the most pressing open questions regarding the flow construct.

First, how (if at all) do the nine items that make up the flow construct hang together? In particular, do any of these items display significant clustering, and if so, what conditions produce this clustering? Further, is it possible to manipulate the phenomenology of flow by manipulating by degree the common preconditions of a flow state?

Second, what is the phenomenological relationship between absorption, effortlessness, action/awareness merging, self-consciousness, sense of control, time distortion, and positive experiential valence? Are some of these aspects of the same mental state, or products of the same process or mechanism, or are flow states characterized by a range of separable mental states related to these differential aspects of phenomenology?

Third, what is the relationship between flow and performance enhancement? Further, might any of the aspects of the flow state individually contribute to performance? If so, why-in what tasks do different elements of flow provide assistance?

\section{Locating flow: a proposal}

I have made a case for the claim that a clear understanding of the flow state's structure and function faces several pressing questions. I now wish to build towards a proposal for addressing these questions. The proposal is that the questions I have articulated are best answered by integrating flow within broader streams of research on the dynamics of conscious thought. To motivate this proposal, I need to briefly discuss the research streams I have in mind - there are three-and how they might be connected.

\subsection{Stream one: spontaneous thought}

The first stream I have in mind concerns the attempt to locate forms of spontaneous thought such as mind wandering, hallucination, and dreaming (Callard et al., 2013; Seli et al., 2018; Smallwood, 2013) within a broader dynamic space of thoughttypes including perseverative thought (ruminations) (Nolen-Hoeksema et al., 2008) and controlled or goal-directed thought (Botvinick et al., 2001). ${ }^{3}$

\footnotetext{
3 Although philosophers have paid little attention to this burgeoning area, recently a few philosophers have begun to make important contributions to this literature (Carruthers, 2018; Dorsch, 2015; Irving, 2016; Irving \& Glasser, 2020; Metzinger, 2018; Murray \& Krasich, 2020; Shepherd, 2019; Sripada, 2018).
} 


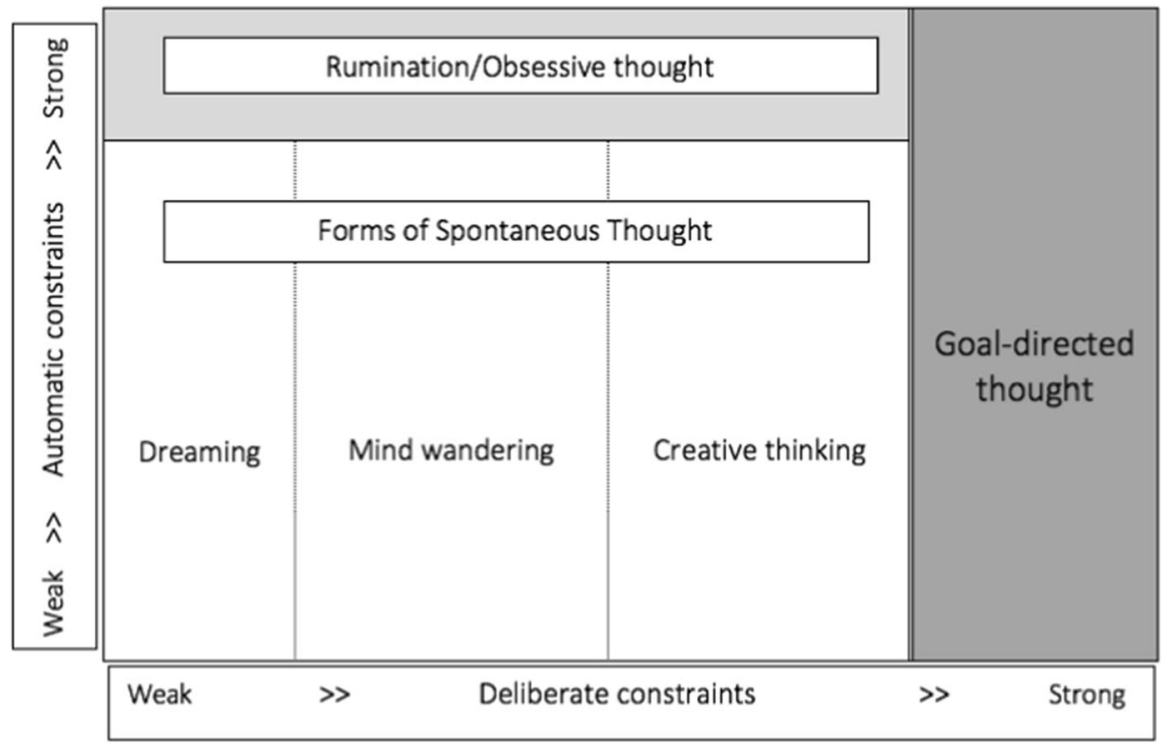

Fig. 1 A conceptual map of the dynamics of thought, proposed by Christoff et al. (2016)

Christoff et al. (2016) propose a framework for organizing the dynamics of thought. Their framework maps thought-types along two dimensions, both of which they spell out in terms of constraints on thought. One dimension involves automatic constraints - constraints imposed on thought by systems that 'operate outside of cognitive control' (719). Affective and sensory salience networks, for example the dorsal attentional network, are their chief example of such constraints, and they hypothesize that depressive and ruminative thought is largely driven by these networks:

People with depression experience thoughts that tend to be inflexible, perseverative and characterized by excessively self-focused, mostly negative content. Rumination is largely involuntary: individuals with depression may want to stop themselves from ruminating but are often unable to do so, suggesting that the constraints on thought in rumination are primarily automatic (725).

By contrast, deliberate constraints are imposed on thought by thought itself, so to - that is, imposed by cognitive control mechanisms. Such constraints include goals and intentions that the individual explicitly sets, within the context of activities of which the individual is aware. 'For example,' they write, 'we can deliberately maintain our attention on a dry and boring lecture, bringing our thoughts back to the lecture whenever they begin to stray' (719) (Fig. 1).

One promising aspect of Christoff et al. proposal is that they are able to link variations in these two dimensions to interactions between broad-scale architectural networks in the brain. They hypothesize, for example, that in spontaneous thought the relative absence of automatic constraints (e.g., salience network activity) and deliberate constraints (e.g., fronto-parietal control network activity), the default 
mode network (especially medial temporal lobe activity) is able to exert 'a relatively strong diversifying influence on the stream of thought' (725, Fig. 4). In perseverative, internally oriented thought, salience networks in tandem with part of the default mode network exert strong automatic constraints on thought. And in goal-directed thought, the fronto-parietal control network dominates, while salience networks and the diversifying influence of the default mode network are relatively muted.

Another promising aspect of this proposal, for present purposes, is that some of the questions it generates enable closer study of aspects of the phenomenology of thought. Christoff et al. call for a more rigorous neurophenomenological approach 'that combines online experience sampling or first-person measures of ongoing thought dynamics with measures of neural activity' (728). As Christoff et al. are aware, much more work is required to understand details regarding shifts in automatic and deliberate constraints, regarding interactions between control and salience networks in the brain, and regarding changes in broad phenomenological patterns.

\subsection{Stream two: cognitive control resource allocation}

The second relevant research stream concerns cognitive control resource allocation. Guiding ideas for this work are that cognitive control is resource limited, and that a cognitive control system that approximates practical wisdom by efficiently distributing resources will do better. One promising line of work explores how the cognitive control system approximates practical wisdom by modelling the decision process that leads to resource distribution using cost-benefit computations (Kool \& Botvinick, 2018; Kool et al., 2017).

According to the Expected Value of Control framework (Botvinick \& Braver, 2015; Kool \& Botvinick, 2018; Shenhav et al., 2017), the cognitive control system monitors the identity and intensity of available 'control signals', and seeks to measure the likelihood of reward given a specification of a control signal along these two dimensions. In addition to the probability of reward, various costs have to be taken into account-including the costs of exerting control at all. In the end, a good cognitive control system is one that approaches optimality at taking the actions most likely to generate positive reward/cost balances. ${ }^{4}$

How do these rewards and costs impact consciousness? This is a vexed question, and there is little consensus on it at present. But an important theme in recent research revolves around the claim that the costs of cognitive control often show up as an aversive experience-the experience of mental effort. According to many, mental effort is familiar in everyday life, ubiquitous across multiple variations of task and circumstance, and integral to cost/benefit computations that are themselves central to the cognitive control system's internal allocation of resources (Kurzban et al., 2013; Kurzban et al., 2013; Shenhav et al., 2017; Kool \& Botvinick, 2018). This is not to deny that other experiences are important-fatigue, anxiety, boredom,

\footnotetext{
${ }^{4}$ For promising work that models cognitive control in terms of cost/benefit computations, see Lieder et al. (2018); Lieder \& Griffiths (2020).
} 
and background affective states likely play important roles (Inzlicht et al., 2015), but how they interact with effort is not well-established.

Might this dynamic range of conscious experiences bear relationships to the experiences characteristic of flow? How, after all, is cognitive control allocated during flow? Notice that the experiences that take predominance in the resource allocation literature are broadly negative, while those that take predominance in the flow literature are said to be positive. I return to this difference below.

\subsection{Stream three: creative thought}

A third stream of research explores coupling between goal-directed and less constrained thought. This is work that uses resting-state and task-based fMRI to explore the neural properties of improvisational action-in, for example, musicians and composers-as well as creative thought (Carruthers, 2018; Limb \& Braun, 2008; Beaty et al., 2016, 2019; Li et al., 2017). Such work has found evidence of key interactions between the default mode network and the fronto-parietal control network in instances of creative thought. For example, highly creative people display greater coupling between these two brain networks (Beaty, 2015). And tasks that call for creative or divergent thinking produce greater coupling between these networks than tasks that do not (Shi et al., 2018). Beaty et al. (2016)—see also Beaty et al. (2019)_review a wide range of work using task-based and resting-state fMRI, and ultimately propose a functional characterization of the coupling that underlies creative thought.

We suggest that the default network contributes to the generation of candidate ideas (potentially useful information derived from long-term memory) in light of its role in self-generated cognition (e.g., episodic memory). Yet, the control network is often required to evaluate the efficacy of candidate ideas and modify them to meet the constraints of task-specific goals. Our model builds on a seminal theory (Jung et al. 2013) that suggested that the default and control networks contribute to idea generation and selection, respectively $(2016,88)$.

\subsection{Connections between streams}

There are several connections between aspects of these research streams-some nascent, some under development. I will briefly discuss three examples.

First, a recent proposal by Girn et al. (2020) connects work on the dynamics of spontaneous thought with work on creative thought. Girn et al. consider the possibility that some activities or modes of thought are characterized by dynamic movement across the two-dimensional space Christoff et al. (2016) map. In particular, they propose that creative thought is characterized by shifts in automatic and deliberate constraints over time as progress in particular tasks is made. What this means is that creative thought may sometimes be characterized by low deliberate or automatic constraints, and sometimes by high constraints, as agents iteratively focus their thoughts based upon how the work is going. In order to understand creative thought, we may need to understand how creative thought unfolds 
over time, and how as it does, the mechanisms that underlie cognitive control exert differential influences on the agent's behavior.

Second, a recent paper by Sripada (2018) forges a connection between work on mind wandering and work on cognitive control resource allocation. Sripada (2018) characterizes mindwandering as an exploratory activity, possibly driven by the default mode network, to be contrasted with the exploitative activity of goal-directed thinking, which is possibly driven by the fronto-parietal control network. How are transitions between these two types of activity managed? Sripada discusses three possible (non-exclusive) regulatory strategies. Let us consider his third. According to Sripada,

It is plausible that goal-directed thinking and mind-wandering exhibit different profiles of marginal utility with time. Goal-directed thinking is likely to exhibit diminishing marginal utility over relatively short stretches of time (i.e., minutes and hours). This is because this mode of thinking is usually initiated to achieve some well-defined specific goal, perhaps deliberating on a set of options or trying to solve some specific problem (e.g., figuring out the shortest route between two locations). After a certain amount of time, additional efforts in deliberating or explicit problem-solving are unlikely to yield additional gains; the person is better off stopping and moving forward with action. Mind-wandering, in contrast, is likely to exhibit relatively constant value over time. (28)

Because of this, the mechanisms that control resource allocation may, in the right conditions, promote shifts between goal-directed thinking and mindwandering. Sripada here refers to a role for cognitive fatigue: 'cognitive fatigue during goaldirected thinking represents the opportunity cost of an alternative mode of serial thought that one could instead be engaged in, an exploratory mode of thought that delivers a more or less stable quantity of utility irrespective of duration' (29).

Traversing a similar space, Shepherd (2019) proposes a slightly different connection between mind wandering and cognitive control. As Shepherd has it,

Mind wandering is caused by the cognitive control system precisely when, and because, the expected value of whatever the agent is doing-usually, exercising control towards the achievement of some occurrent goal-is deemed too low, and this "too low" judgment generates a search for a better goal, or task (5).

There are important differences between Shepherd's and Sripada's proposal. Shepherd has mindwandering as, sometimes, itself a cognitive control task. Sripada has it as being driven by fatigue. Both, then, make predictions about how the cognitive control networks involved would behave. These predictions can be addressed in experimental paradigms that look at goal-directed thought and mindwandering simultaneously-paradigms that explore connections between, and perhaps dynamic transitions between, these modes of thinking.

Further exploration of these projects is not the main goal of this paper. I am interested in how further connections might be forged between flow and these three 


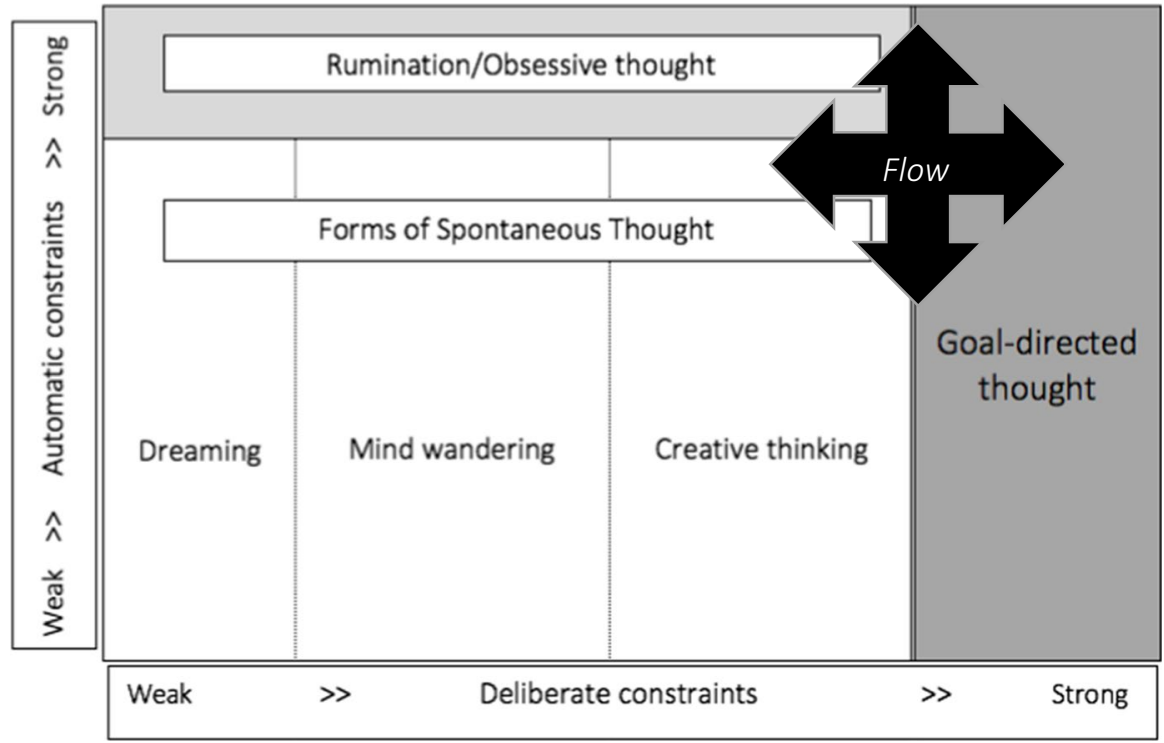

Fig. 2 A possible location for flow states in Christoff et al. (2016) map. Open questions include the importance of automatic constraints for flow, whether and how often flow shows up in types of creative thinking, or in obsessive thinking, and whether additional dimensions-concerning skill/difficulty balance, or concerning individual skills that permit easier entry into the flow state-are required to accurately map the flow state's dynamics

streams. One might try to conceptualize this within the map provided by Christoff et al., for one natural thought is that the flow state occupies the space where goaldirected constraints are relatively high. Of course, as I have tried to make clear in this paper, open questions about the behavior of the flow state in relation to nearby modes of thought remain. To what extent do automatic constraints play a role in flow entry and exit? Further, are the two dimensions Christoff et al. map sufficient to capture flow state dynamics? Given the emphasis many place on skill/difficulty balance, and given the possible importance of individual-level differences in attentional capacities, background knowledge of the task, and more, it may be that additional dimensions are required to map flow state dynamics (Fig. 2).

\section{Locating flow in the dynamics of thought}

Thus far, I have raised three sets of questions about the flow construct, and I have discussed three research streams that can, I claim, bear fruitfully on these questions. Of course the direction of influence might go both ways. It might be that we can better understand thought dynamics, cognitive control resource allocation, or creative thought by considering flow alongside them (for an interesting paper exploring flow alongside perseverative thought, see Hupfeld et al., 2019). But let us keep the focus on the questions I have articulated for the moment. How might we better understand flow by studying it in the context of these research streams? 
The first set of questions I raised about the flow construct concerned the way that the nine items that make up the flow construct hang together-to what extent (and in what conditions) they correlate, and why. One important aspect of any examination of these questions concerns the presence or absence of these items in 'nearby', non-flow states. To what extent do we find the sense of control, action/awareness merging, attentional absorption, or a sense of intrinsic reward in instances of creative thought, or in non-flow cognitive control conditions as occur in standard tasks like go/no-go or a Stroop task? This could be studied by simply using paradigms prevalent in work on creative thinking (e.g., the alternate uses divergent thinking task) and on cognitive control (e.g., task switching) while measuring the prevalence of flow items. Or one might think more creatively, utilizing naturalistic cognitive control tasks in conjunction with experience sampling, or qualitative interviews as in many studies of flow in sport psychology. Understanding how flow items appear and correlate across a broader dynamic space will help us understand how their presence in flow may (or may not) be distinct or unusual.

Moreover, understanding how these items might be manipulated by degree across changing conditions should form an important part of an account of flow. Csikszcentmihalyi et al. sometimes characterize the dynamics of flow as driven by its inherently rewarding phenomenology, and by the negative phenomenology of nearby states. For example, at the margins of the flow state-hypothesized to occur because of changes to the skill/difficulty balance-we are said to find experiences of anxiety or boredom.

The balance is intrinsically fragile. If challenges begin to exceed skills, one first becomes vigilant and then anxious; if skills begin to exceed challenges, one first relaxes and then becomes bored. Shifts in subjective state provide feedback about the changing relationship to the environment. Experiencing anxiety or boredom presses a person to adjust his or her level of skill and/or challenge in order to escape the aversive state and reenter flow (Csikszcentmihalyi et al., 2014, 232).

In this connection, recall Girn et al. proposal regarding the dynamics of creative thought. Might a similar dynamics be found for entry into and exit from a flow state? The characterization of flow as requiring clear goals and clear feedback suggests that flow is easier to achieve when deliberate constraints are relatively high - a prediction that follows from this is that creative thought would incorporate aspects of flow to the extent that deliberate constraints are high (provided that other constraints, e.g., clear feedback, are met) (see Biasutti \& Frezza, 2009). A further prediction one might make here is that flow states during creative thought would be associated with greater coupling between DMN and the fronto-parietal control network (for provisional evidence in this direction, see Ulrich et al., 2018). Note, in this connection, that Beaty et al. (2016) have speculated that the degree to which a task is goal-directed may impact the nature of the DMN-fronto-parietal network coupling, with clearer coupling in the case of relatively clear goals, and less cooperation 'in the absence of a clear task goal or when top-down constraints are relaxed' $(2016,93)$. 
A second set of questions I articulated concerned the phenomenology of the flow state. Here there is an interesting tension between work on flow and work on cognitive control resource allocation. Flow is highly goal-directed, and thus involves cognitive control, but is also said to be both effortless-a "peak attentional experience' - and inherently rewarding. This sits at odds with most work on cognitive control resource allocation, which is often assumed to be inherently aversive, and effortful. There is a possibility here of plugging a significant gap-what we might think of as the positive experiential side of cognitive control. Is it the case that the flow state is best described as effortless, or might effort be present to some degree in the flow state? Does effort only emerge when agents transition out of a flow state? Is effort intrinsically aversive, or might the phenomenology of effort be more complex? Note, in this connection, that Székeley and Michael (2020) have recently proposed that effort may at times be negative and at times neutral or positive. The question needs to be researched, but it may be that the conditions of flow provide a unique testing ground for such a view regarding the valence of mental effort.

One basic finding in the cognitive control literature is that as agents remain on task, performance tends to decrease. This often produces a characteristic performance curve, and some have sought to explain this curve by reference to feelings of effortfulness or fatigue (Kurzban et al., 2013; Sripada, 2018). A natural question concerns performance decrements in flow-like conditions. How, for example, does flow relate to vigilance and fatigue? Do we see the same performance curves over time for tasks that tend to produce flow? Might we find an increase in effortfulness over (similar) time periods, even in flow-like conditions, or might the rewarding nature of flow tasks produce different performance curves and different reports of effortfulness? If so, many of the models that drive theorizing in the cognitive control literature will need to accommodate this fact.

These questions might be explored in paradigms that 'gamify' cognitive control. Many video games, for example, essentially embed iterated cycles of cognitive control operations, asking players to rapidly switch tasks, and to engage in go/ no-go like tasks, with reward and punishment embedded as well. It might be possible to explore the phenomenology and performance curves associated with flow by using (perhaps by creating — see Anguera \& Gazzaley, 2015; Leider \& Griffiths, 2016) games that embed cognitive control operations while at the same time producing flow-like conditions. Promisingly, Huskey et al. (2018) have developed an open source video game for just this purpose, finding that increased reports of intrinsic reward correlated with increased attentional engagement on the task.

Further aspects of the flow state might be important for understanding cognitive control more broadly. For example, the sense of control seems (to me) to be an important aspect of the phenomenology of thought (see Mylopoulos \& Shepherd, 2020). It would be interesting to know if the sense of control varies across states of flow, creative thought, and more standard cognitive control conditions. Additionally, since it is plausible to think of the sense of control as a metacognitive feeling (Proust, 2013), it would be useful to know to what extent the sense of control is accurate in flow conditions, and whether this differs from non-flow conditions.

A third set of questions I raised regarding flow concerned the relationship between flow and performance. I argued that an understanding of the flow-performance 
relationship faces a difficult confound, since the conditions that might produce flow might also be largely responsible for any enhanced performance as well. I also argued that success in real-world conditions might require entry into and exit from flow-like conditions, depending upon circumstances. One interesting question here, however, is how ease of entry into and exit from a flow state is related to performance. An interesting hypothesis is that, while flow itself is not important for performance enhancement, the ability to enter flow and disengage from flow more readily is associated with performance enhancement.

\section{Conclusion}

I have raised several questions about the flow construct, and argued that these questions are best pursued by research that studies flow alongside nearby modes of thought-mindwandering, creative thought, perseverative thought, and goal-directed thought more broadly. Others have made tentative steps in connecting these various areas of research. My aim here has been to make relevant questions and connections more explicit, in order to motivate the kind of research that could answer the questions I have articulated. One possible upshot of this kind of research could be data that helps bring theoretical integration between research streams that often utilize very different methodologies and focus on different aspects of mental life.

In addition, I have suggested that studying flow in this way offers a possibility of plugging a significant gap: the positive experiential (and motivational) side of cognitive control resource allocation. Studying the rewarding nature of aspects of goal-directed thinking may afford additional insights into the mechanisms that drive resource allocation, and may push us towards a more thorough account of the experiential space surrounding thought.

Acknowledgements A meeting of CIFAR's Mind, Brain, and Consciousness group spurred this paper into existence: thanks to attendees of that meeting for comments and encouragement. Thanks also to Myrto Mylopoulos for conversation about the paper, and to referees for constructive comments.

Funding The research was supported by ERC Starting Grant 757698, awarded under the Horizon 2020 Research and Innovation Programme, and also by an Azrieli Global Scholar fellowship provided by the Canadian Institute for Advanced Studies program on Mind, Brain, and Consciousness.

Open Access This article is licensed under a Creative Commons Attribution 4.0 International License, which permits use, sharing, adaptation, distribution and reproduction in any medium or format, as long as you give appropriate credit to the original author(s) and the source, provide a link to the Creative Commons licence, and indicate if changes were made. The images or other third party material in this article are included in the article's Creative Commons licence, unless indicated otherwise in a credit line to the material. If material is not included in the article's Creative Commons licence and your intended use is not permitted by statutory regulation or exceeds the permitted use, you will need to obtain permission directly from the copyright holder. To view a copy of this licence, visit http://creativecommons.org/licen ses/by/4.0/. 


\section{References}

Aherne, C., Moran, A. P., \& Lonsdale, C. (2011). The effect of mindfulness training on athletes flow: An initial investigation. The Sport Psychologist, 25(2), 177-189.

Anguera, J. A., \& Gazzaley, A. (2015). Video games, cognitive exercises and the enhancement of cognitive abilities. Current Opinion in Behavioral Sciences, 4, 160-165.

Beaty, R. E. (2015). The neuroscience of musical improvisation. Neuroscience \& Biobehavioral Reviews, $51,108-117$.

Beaty, R. E., Benedek, M., Silvia, P. J., \& Schacter, D. L. (2016). Creative cognition and brain network dynamics. Trends in Cognitive Sciences, 20(2), 87-95.

Beaty, R. E., Seli, P., \& Schacter, D. L. (2019). Network neuroscience of creative cognition: Mapping cognitive mechanisms and individual differences in the creative brain. Current Opinion in Behavioral Sciences, 27, 22-30.

Biasutti, M., \& Frezza, L. (2009). Dimensions of music improvisation. Creativity Research Journal, 21(2-3), 232-242.

Bishop, M. A. (2015). The good life: Unifying the philosophy and psychology of well-being. USA: Oxford University Press.

Botvinick, M., \& Braver, T. (2015). Motivation and cognitive control: From behavior to neural mechanism. Annual Review of Psychology, 66, 83-113.

Botvinick, M. M., Braver, T. S., Barch, D. M., Carter, C. S., \& Cohen, J. D. (2001). Conflict monitoring and cognitive control. Psychological Review, 108(3), 624.

Boyd, R. (1991). Realism, anti-foundationalism and the enthusiasm for natural kinds. Philosophical Studies, 61(1), 127-148.

Bühlmayer, L., Birrer, D., Röthlin, P., Faude, O., \& Donath, L. (2017). Effects of mindfulness practice on performance-relevant parameters and performance outcomes in sports: A meta-analytical review. Sports Medicine, 47(11), 2309-2321.

Callard, F., Smallwood, J., Golchert, J., \& Margulies, D. S. (2013). The era of the wandering mind? Twenty-first century research on self-generated mental activity. Frontiers in Psychology, 4, 891.

Carruthers, P. (2018). Mechanisms for constrained stochasticity. Synthese

Chavez, E. J. (2008). Flow in sport: A study of college athletes. Imagination Cognition and Personality, 28(1), 69-91.

Christoff, K., Irving, Z. C., Fox, K. C., Spreng, R. N., \& Andrews-Hanna, J. R. (2016). Mind-wandering as spontaneous thought: A dynamic framework. Nature Reviews Neuroscience, 17(11), 718-731.

Cochrane, T. (2017). Group flow. In M. Lesaffre, P.-J. Maes, \& M. Leman (Eds.), The routledge companion of embodied music interaction (pp. 133-140). London: Routledge.

Csikszcentmihalyi, M. (2014). Play and intrinsic rewards. In Flow and the foundations of positive psychology (pp. 135-154). Springer, Dordrecht.

Csikszentmihalyi, M., Abuhamdeh, S., \& Nakamura, J. (2005). Flow. In A. J. Elliot \& C. S. Dweck (Eds.), Handbook of competence and motivation (pp. 598-608). Guilford.

Csikszcentmihalyi, M., Abuhamdeh, S., \& Nakamura, J. (2014). Flow. In Flow and the foundations of positive psychology (pp. 227-238). Springer.

De Manzano, Ö., Theorell, T., Harmat, L., \& Ullén, F. (2010). The psychophysiology of flow during piano playing. Emotion, 10(3), 301.

Debus, D. (2015). Losing oneself in a good way: On the value of full attention. European Journal of Philosophy, 23(4), 1174-1191.

Dorsch, F. (2015). Focused daydreaming and mind-wandering. Review of Philosophy and Psychology, 6(4), 791-813.

Dreyfus, H. L. (2005). Overcoming the myth of the mental: How philosophers can profit from the phenomenology of everyday expertise. In Proceedings and addresses of the American Philosophical Association (pp. 47-65). American Philosophical Association.

Girn, M., Mills, C., Roseman, L., Carhart-Harris, R. L., \& Christoff, K. (2020). Updating the dynamic framework of thought: creativity and psychedelics. NeuroImage, 213, 116726.

Harris, D., Allen, K., Vine, S. J., \& Wilson, M. (2020). A systematic review and meta-analysis of the relationship between flow states and performance. https://doi.org/10.31234/osf.io/qg852

Hupfeld, K. E., Abagis, T. R., \& Shah, P. (2019). Living in the zone: hyperfocus in adult ADHD. ADHD Attention Deficit and Hyperactivity Disorders, 11(2), 191-208. 
Huskey, R., Craighead, B., Miller, M. B., \& Weber, R. (2018). Does intrinsic reward motivate cognitive control? A naturalistic-fMRI study based on the synchronization theory of flow. Cognitive, Affective, \& Behavioral Neuroscience, 18(5), 902-924.

Inzlicht, M., Bartholow, B. D., \& Hirsh, J. B. (2015). Emotional foundations of cognitive control. Trends in Cognitive Sciences, 19(3), 126-132.

Irving, Z. C. (2016). Mind-wandering is unguided attention: accounting for the purposeful wanderer. Philosophical Studies, 173(2), 547-571.

Irving, Z. C., \& Glasser, A. (2020). Mind-wandering: A philosophical guide. Philosophy Compass, 15(1), e12644.

Jackson, S. A., Ford, S. K., Kimiecik, J. C., \& Marsh, H. W. (1998). Psychological correlates of flow in sport. Journal of Sport and Exercise Psychology, 20(4), 358-378.

Jackson, S. A., \& Marsh, H. W. (1996). Development and validation of a scale to measure optimal experience: The flow state scale. Journal of Sport and Exercise Psychology, 18(1), 17-35.

Jung, R. E., Mead, B. S., Carrasco, J., \& Flores, R. A. (2013). The structure of creative cognition in the human brain. Frontiers in Human Neuroscience, 7. https://doi.org/10.3389/fnhum.2013.00330

Kennedy, P., Miele, D. B., \& Metcalfe, J. (2014). The cognitive antecedents and motivational consequences of the feeling of being in the zone. Consciousness and Cognition, 30, 48-61.

Kool, W., Shenhav, A., \& Botvinick, M. M. (2017). Cognitive Control as Cost-Benefit Decision Making. The Wiley Handbook of Cognitive Control (pp. 167-189)

Kool, W., \& Botvinick, M. (2018). Mental labour. Nature Human Behaviour, 2(12), 899-908.

Kurzban, R., Duckworth, A., Kable, J. W., \& Myers, J. (2013). An opportunity cost model of subjective effort and task performance. Behavioral and Brain Sciences, 36(6), 661-679.

Li, J., Zhang, D., Liang, A., Liang, B., Wang, Z., Cai, Y., \& Liu, M. (2017). High transition frequencies of dynamic functional connectivity states in the creative brain. Scientific Reports, 7(1), 1-12.

Lieder F., Griffiths T.L. (2016). Helping people make better decisions using optimal gamification. In A Papafragou, D Grodner, D Mirman, JC Trueswell (Eds.) Proceedings 38th Annual Conference Cognitive Science Society, Philadelphia, pp. 2075-80, Austin

Lieder, F., \& Griffiths, T. L. (2020). Resource-rational analysis: understanding human cognition as the optimal use of limited computational resources. Behavioral and Brain Sciences, 43, e1.

Lieder, F., Shenhav, A., Musslick, S., \& Griffiths, T. L. (2018). Rational metareasoning and the plasticity of cognitive control. PLoS Computational Biology, 14(4), e1006043.

Limb, C. J., \& Braun, A. R. (2008). Neural substrates of spontaneous musical performance: An fMRI study of jazz improvisation. PLoS ONE, 3(2), e1679.

Marty-Dugas, J., \& Smilek, D. (2019). Deep, effortless concentration: Re-examining the flow concept and exploring relations with inattention, absorption, and personality. Psychological Research Psychologische Forschung, 83(8), 1760-1777.

Metzinger, T. (2018). Why is mind wandering interesting for philosophers. The Oxford handbook of spontaneous thought: Mind-wandering, creativity, and dreaming pp. 97-111.

Montero, B. (2016). Thought in action: Expertise and the conscious mind. Oxford: Oxford University Press.

Murray, S., \& Krasich, K. (2020). Can the mind wander intentionally? Mind \& Language. https://doi.org/ $10.1111 /$ mila. 12332

Mylopoulos, M., \& Shepherd, J. (2020). The experience of agency. In U. Kriegel (Ed.), The oxford handbook of the philosophy of consciousness. Oxford: Oxford University Press.

Nakamura, J., \& Csikszentmihalyi, M. (2014). The concept of flow. In Flow and the foundations of positive psychology (pp. 239-263). Springer, Dordrecht.

Nolen-Hoeksema, S., Wisco, B. E., \& Lyubomirsky, S. (2008). Rethinking rumination. Perspectives on Psychological Science, 3(5), 400-424.

Proust, J. (2013). The philosophy of metacognition: Mental agency and self-awareness. Oxford: OUP.

Reber, R., Schwarz, N., \& Winkielman, P. (2004). Processing fluency and aesthetic pleasure: Is beauty in the perceiver's processing experience? Personality and Social Psychology Review, 8(4), 364-382.

Scott-Hamilton, J., Schutte, N. S., \& Brown, R. F. (2016). Effects of a mindfulness intervention on sportsanxiety, pessimism, and flow in competitive cyclists. Applied Psychology Health and Well-Being, $8(1), 85-103$.

Seli, P., Kane, M. J., Smallwood, J., Schacter, D. L., Maillet, D., Schooler, J. W., \& Smilek, D. (2018). Mind-wandering as a natural kind: A family-resemblances view. Trends in Cognitive Sciences, 22(6), 479-490. 
Shenhav, A., Musslick, S., Lieder, F., Kool, W., Griffiths, T. L., Cohen, J. D., \& Botvinick, M. M. (2017). Toward a rational and mechanistic account of mental effort. Annual Review of Neuroscience, 40, 99-124. https://doi.org/10.1146/annurev-neuro-072116-031526

Shepherd, J. (2019). Why does the mind wander? Neuroscience of Consciousness, 2019(1), niz014.

Shi, L., Sun, J., Xia, Y., Ren, Z., Chen, Q., Wei, D., \& Qiu, J. (2018). Large-scale brain network connectivity underlying creativity in resting-state and task fMRI: cooperation between default network and frontal-parietal network. Biological Psychology, 135, 102-111.

Smallwood, J. (2013). Distinguishing how from why the mind wanders: a process-occurrence framework for self-generated mental activity. Psychological Bulletin, 139(3), 519.

Sripada, C. S. (2018). An exploration/exploitation trade-off between mind wandering and goal-directed thinking. The Oxford handbook of spontaneous thought: Mind-wandering, creativity, and dreaming pp. 23-34

Swann, C., Crust, L., Jackman, P., Vella, S. A., Allen, M. S., \& Keegan, R. (2017). Psychological states underlying excellent performance in sport: Toward an integrated model of flow and clutch states. Journal of Applied Sport Psychology, 29(4), 375-401.

Swann, C., Keegan, R. J., Piggott, D., \& Crust, L. (2012). A systematic review of the experience, occurrence, and controllability of flow states in elite sport. Psychology of Sport and Exercise, 13(6), 807-819.

Székely, M., \& Michael, J. (2020). The sense of effort: A cost-benefit theory of the phenomenology of mental effort. Review of Philosophy and Psychology. https://doi.org/10.1007/s13164-020-00512-7

Ulrich, M., Niemann, J., Boland, M., Kammer, T., Niemann, F., \& Grön, G. (2018). The neural correlates of flow experience explored with transcranial direct current stimulation. Experimental Brain Research, 236(12), 3223-3237.

Vuorre, M., \& Metcalfe, J. (2016). The relation between the sense of agency and the experience of flow. Consciousness and Cognition, 43, 133-142. 